\title{
The Evaluation of Neutrophil to Lymphocyte Ratio in Kawasaki Disease and Its Value in Predicting IVIG Therapy Resistance
}

\section{Hamidreza Houshmand}

Urmia University of Medical Sciences

Ramin Farhadi

Urmia University of Medical Sciences

Amin Sedokani ( $\sim$ a.sedokani@gmail.com )

Urmia University of Medical Sciences https://orcid.org/0000-0003-1325-3309

\section{Research Article}

Keywords: Kawasaki, IVIG, Neutrophil-Lymphocyte Ratio

Posted Date: July 27th, 2021

DOl: https://doi.org/10.21203/rs.3.rs-725186/v1

License: (1) This work is licensed under a Creative Commons Attribution 4.0 International License.

Read Full License 


\section{Abstract}

Introduction: Kawasaki disease is a common systemic vasculitis of unknown cause in children and involves various organs but the main damage of this multisystemic vasculitis is mainly in the coronary arteries. Currently, the main treatments for this disease include aspirin therapy and IVIG. In the present study, we investigated the ratio of neutrophils to lymphocytes in patients with Kawasaki disease and its relationship with IVIG resistance in Kawasaki patients.

Method: First, we reviewed the clinical records of patients diagnosed with Kawasaki, who received treatment at Motahari Hospital in Urmia from 2008 to 2018 (IVIG therapy). We re-examined Kawasaki diagnostic criteria for each individual before enrolling patients.

Results: In our study, initially 460 cases of patients who were admitted to Motahari Hospital in Urmia between 2009 and 2019 with an initial diagnosis of Kawasaki disease were reviewed. Among them, patients who met Kawasaki diagnostic criteria were included in the final review and the rest of the cases were excluded from the study according to the exclusion criteria. Finally, out of 460 cases, 241 patients were included in the final analysis. The results indicated high blood leukocyte count ( $p$-value $=0.013)$ and low urinary leukocyte count ( $\mathrm{p}$-value $=0.01$ ) were directly related to response to treatment in Kawasaki patients, but age, sex, neutrophil ratio to Lymphocytes, neutrophil count, blood lymphocytes, CRP, hypoalbuminemia, ALT levels, anemia, thrombocytosis, and the time interval between onset of symptoms and treatment response to IVIG therapy in Kawasaki patients are not significantly related.

Conclusion: High blood leukocyte count and low urinary leukocyte count in patients can be good criteria for predicting the prognosis of Kawasaki patients in response to IVIG, while the ratio of neutrophils to lymphocytes is not effective in this prediction.

\section{Key Messages}

- In the present study, we investigated the ratio of neutrophils to lymphocytes (as an available inflammatory marker) in patients with Kawasaki disease and its relationship with IVIG resistance in hospitalized patients. 241 patients with the diagnosis of Kawasaki disease were reviewed.

- The high blood leukocyte count $(p$-value $=0.013)$ and low urinary leukocyte count $(p$-value $=0.01)$ were directly related to response to treatment in Kawasaki patients.

- Age, sex, neutrophil to Lymphocytes ratio, neutrophil count, blood lymphocytes, CRP, hypoalbuminemia, ALT levels, anemia, thrombocytosis, and the time interval between onset of symptoms and treatment response to IVIG therapy in Kawasaki patients are not significantly related to response to treatment.

\section{Introduction}


Kawasaki disease is a common systemic vasculitis of unknown cause in children that is seen in all parts of the world, including our country. During this skin disease, mucous membranes of the whole body, lymph nodes, meninges, intestines, liver, gallbladder, joints, genitals and heart are involved. But the major damage of this multisystemic vasculitis is mainly in the coronary arteries ${ }^{1}$. In the last decade, Kawasaki disease has been the most common heart disease in developed countries and its prevalence is increasing 2,3 . The disease is mainly (more than $85 \%$ ) in children aged one to five years and outside this age range, the disease occurs atypically ${ }^{4}$. The disease mainly presents as a self-limiting condition that includes a fever and inflammatory manifestations that resolve spontaneously for up to 12 days; however, complications such as coronary artery aneurysms, decreased myocardial contractility, heart failure, myocardial infarction, arrhythmias, and peripheral artery occlusion are some of the complications of this disease that can lead to morbidity and high mortality. The clinical complications of Kawasaki disease are due to systemic inflammation in the medium-size arteries and the diagnosis will be based on the symptoms of systemic inflammation such as fever and the symptoms of cutaneous mucositis. Clinical manifestations of this disease, at first include an initial period including gastrointestinal and respiratory symptoms and then with non-purulent conjunctivitis, red lips and oral mucosa, changes in the upper gastrointestinal tract epithelium, skin polymorphic rashes, changes in the fingers and cervical lymphadenopathy. The cardiac complications of this disease start mostly subclinical and will appear as myocardial infarction and sudden death years after recovery ${ }^{5-7}$. Treatment with high doses of intravenous immunoglobulin (IVIG) in the first ten days after the onset of symptoms can significantly prevent coronary aneurysms. Currently, the main treatments for this disease include aspirin therapy and IVIG. However, 10 to $20 \%$ of children patients do not respond to this treatment ${ }^{8}$. So far, several scoring scales have been designed to predict IVIG response in Japan, while these scales do not seem to be of much value in other countries. Alternatively, screening for inflammatory cytokines and surface markers of T cells may be predictive of resistance to IVIG therapy but is not routinely available and requires high costs ${ }^{9}$. Since chronic and uncontrolled inflammation can lead to coronary artery disease and resistance to IVIG treatment, the use of such markers appears to be useful in predicting IVIG resistance. The white blood cell count and its subtypes in the peripheral blood are classic determinants of inflammation. In general, neutrophil counts reflect acute and nonspecific inflammation, while lymphocytes are primarily markers of immune regulation. Therefore, the neutrophils to lymphocytes ratio (NLR) may indicate a balance between acute inflammation and immune regulation ${ }^{10}$. Previous studies have shown that the ratio of neutrophils to lymphocytes is an important determinant of inflammation and can help determine the prognosis of cardiovascular disease and cancer. There have also been reports of a correlation between neutrophil to lymphocyte ratio with surgical stress, systemic inflammation, sepsis, and even the severity of coronary artery disease. Previous studies have shown that the ratio of neutrophils to lymphocytes after initiation of IVIG therapy in Kawasaki patients may be a predictor of resistance to IVIG therapy and the incidence of coronary artery disease ${ }^{11}$. However, there is not much strong evidence in favor of this effect and there are contradictory results between the studies. In the present study, we investigated the ratio of neutrophils to lymphocytes in patients with Kawasaki disease and its relationship with IVIG resistance in patients referred to Motahari Hospital in Urmia. 


\section{Material And Methods}

First, we reviewed the clinical records of patients diagnosed with Kawasaki, who received treatment at Motahari Hospital in Urmia from 2009 to 2019 (IVIG therapy). We re-examined Kawasaki diagnostic criteria for each individual prior entering the patient to the study. Kawasaki diagnostic criteria were as follows: Bilateral non-purulent conjunctivitis, change in one of the mucous membranes of the upper respiratory tract, including pharyngeal congestion, dry and cracked lips, congested lips and strawberry tongue, one or more changes in the ends including peripheral erythema, peripheral edema, scaling around the nails and pervasive scaling, Polymorphic rash mainly on the trunk, Cervical lymphadenopathy with a diameter of $>1.5 \mathrm{~cm}$. The patient is known as a Kawasaki patient if he/she has 4 of the above cases with fever for more than 5 days.

Inclusion criteria will be as follows; patients who fill Kawasaki diagnostic criteria, patients who do not meet the diagnostic criteria (have 2 or 3 criteria), have a fever for more than 5 days and have 3 complementary criteria (anemia, thrombocytosis, increased neutrophils, increased liver enzymes, the presence of leukocytes in the urine). Exclusion criteria were non-fulfillment of Kawasaki diagnostic criteria or failure to include the necessary information for study in patients' records, or the cause of fever was due to a disease other than Kawasaki disease.

We also obtained the following information from the laboratory data section of the patient's clinical record and recorded it in the study checklist: neutrophil count, lymphocyte count, neutrophil to lymphocyte ratio, and serum CRP level. Then, based on the response of each patient to treatment, we divided them into two groups of resistant to treatment and response to treatment. The response and resistance to treatment were defined as follows: responsive to treatment: The fever subsides within 24 hours after treatment with no observed recurrence. Resistant to treatment: a patient who still had a fever for more than 24 hours after treatment or a Kawasaki-related recurrent fever that occurs after a period of non-fever.

\section{Data Analysis}

Data were reported as mean and standard deviation or number (percentage) in the form of appropriate tables and graphs. T-test was used to compare the means. Data analysis was performed using SPSS16 software and the significance level was $<0.05$.

\section{Results}

In our study, 460 cases of patients who were admitted to Motahari Hospital of Urmia between 2009 and 2019 with an initial diagnosis of Kawasaki disease were initially examined. Of these, patients who met Kawasaki diagnostic criteria entered the final review and the remaining cases were excluded from the study according to the exclusion criteria. Finally, out of 460 cases, 241 patients were included in the final analysis (Table 1, 2). 
In our study, the mean age was $34.86 \pm 25.6$ months with a minimum age of 2 and a maximum of 132 months. The mean age was $32.62 \pm 24.97$ months in patients who responded well to treatment and $38.75 \pm 26.35$ months in patients who did not respond well to IVIG treatment. In age analysis, age had no significant relationship with response to disease treatment $(p$-value $=0.192)$.

\section{Gender}

The number of male patients compared to female patients in our study was 137 to 104 with a relative prevalence of $56.8-43.2 \%$, respectively. This rate was 85 to 52 in patients who responded well to treatment and 67 to 36 in patients who did not respond well to IVIG treatment, respectively. Statistical analysis showed that the response rate to treatment based on gender in these patients in our study was not significantly different $(p$-value $=0.8)$.

\section{Conjunctivitis}

Among the 241 patients in the study, the presence of conjunctivitis was reported to be positive in 130 (53.9\%) patients with a relative prevalence of. While 110 patients (46.1\%), had no symptoms of conjunctivitis during hospitalization.

\section{Mucosal Membranes Changes}

In this study, we examined changes in mucous membranes including hyperemia or erythema of the throat, dry and cracked lips, lip hyperemia, and strawberry tongue from patients' histories. The results showed that 195 patients (80.9\%) had one of the above symptoms in the history and clinical examinations while 46 patients $(19.1 \%)$ did not have any of the above symptoms.

\section{Changes in Extremes}

This criterion included one or more changes in the extremities, including peripheral erythema, peripheral edema, scaling around the nails, and pervasive scaling. According to our results, only 58 patients (24.1\%), had one or more of these symptoms, and 183 patients (75.9\%), did not have these symptoms.

\section{Polymorphic rash and erythema of the hands and feet}

Polymorphic rashes mainly on the trunk were examined in this study. Of the patients in the study, 117 patients $(48.5 \%)$ had this rash, while 124 patients $(51.5 \%)$, were without this symptom. Erythema in the hands or feet was present in 48 patients (19.9\%) but 193 patients $(80.1 \%)$ did not have this symptom.

\section{Lymphadenopathy}

Cervical lymphadenopathy with a diameter of more than $1.5 \mathrm{~cm}$ was defined as the presence of lymphadenopathy in the disease criteria in this study. In this case, 66 patients $(27.4 \%)$ had cervical 
lymphadenopathy, while 175 patients (72.6\%) had not cervical lymphadenopathy.

\section{Neutrophils to lymphocytes ratio}

In the present study, the mean ratio of neutrophils to lymphocytes in patients who responded to appropriate treatment was $3.06 \pm 2.89$, while in patients who did not respond appropriately to treatment, this ratio was $4 \pm 3.51$. This ratio was not significantly related to the response to IVIG treatment in Kawasaki patients ( $p$-value $=0.058$ ).

\section{Blood neutrophil and lymphocyte count}

The mean number of neutrophils and lymphocytes in our patients was $6286 \pm 1708$ and $3150 \pm 2983$ per $\mathrm{mm}^{3}$, respectively. None of these cells had a significant relationship with the response to IVIG treatment in Kawasaki patients (neutrophil $p$-value $=0.27$, lymphocyte $p$-value $=0.14$ ).

\section{C-Reactive Protein}

The acute phase reactant CRP was quantitatively investigated in the present study. The mean CRP of patients was $27.65 \pm 41.90 \mathrm{mg} / \mathrm{L}$. This rate was $40.79 \pm 26.33 \mathrm{mg} / \mathrm{L}$ in patients with the appropriate response to treatment and $43.84 \pm 29.88 \mathrm{mg} / \mathrm{L}$ in patients with resistance to treatment. Analysis of the results showed that there was no significant difference in CRP level with response to IVIG treatment in Kawasaki patients ( $p$-value $=0.16)$.

\section{Blood leukocytes}

The mean number of leukocytes in patients with an appropriate response to treatment was $14266 \pm 6291$ per $\mathrm{mm}^{3}$ and in patients who were resistant to treatment was $13450 \pm 4443$ per mm3. This rate was significantly different in the responsive to with resistant to IVIG treatment in Kawasaki patients. The number of blood leukocytes in patients who responded to appropriate treatment was significantly higher than patients who were resistant to IVIG treatment $(p-v a l u e=0.013)$.

\section{Hypoalbuminemia}

In the present study, hypoalbuminemia was defined as less than $3 \mathrm{~g} / \mathrm{dl}$, in which 13 patients (5.4\%), had albumin below 3 and 228 patients (94.6\%), had albumin equal to or above $3 \mathrm{~g} / \mathrm{dL}$. Data analysis did not show a significant relationship between hypoalbuminemia and response to treatment in the present study $(p$-value $=0.18)$.

\section{Alanine transaminase}

The level of blood ALT enzyme in patients was adjusted according to the age of patients and was divided into two states, normal and normal in which 46 patients (19.1\%) had elevated ALT enzyme, while 195 patients $(80.9 \%)$ had normal ALT enzyme levels. Data analysis showed that there was no significant relationship between response to treatment and increase in ALT enzyme ( $p$-value $=0.15)$. 


\section{Anemia}

After adjusting the hemoglobin level based on the age of the patients and determining the presence or absence of anemia, the results showed that 182 patients, $(75.5 \%)$ have had anemia and 59 patients $(24.5 \%)$ had normal hemoglobin. Data analysis did not show a significant difference between the presence of anemia and response to treatment in the present study ( $p$-value $=0.15)$.

\section{Thrombocytosis}

Platelets above or equal to 450,000 per $\mathrm{mm}^{3}$ were considered thrombocytosis in the present study, and 91 patients (37.8\%), had thrombocytosis, while 150 patients $(62.2 \%)$, had platelets below 450,000 . Data analysis did not show a significant difference between the presence of thrombocytosis and response to treatment in the present study ( $p$-value $=0.53$ ).

\section{Pyuria}

The presence of leukocytes above 10 per HPF[1] in the urine of patients was considered as the presence of pyuria in the present study. 73 patients $(30.3 \%)$ of patients had pyuria but 168 patients $(69.7 \%)$ of patients without pyuria. Data analysis showed that there is a significant difference between pyuria and response to treatment, so that in patients with leukocytes less than 10 or 10 per urine HPF, the response to treatment was significantly higher than patients with urinary leukocytes per HPF more than 10 ( $p$-value $=0.01)$.

\section{Echocardiographic criteria}

Echocardiographic criteria studied in the present study included LAD or RCA coronary artery transparency with a Z-score between 2 and 2.5, no reduction in vascular diameter, decreased left ventricular function, mitral valve insufficiency and pericardial effusion. There were two criteria of decreased left ventricular function and vascular transparency, each in only 3 patients, which was equivalent to $1.2 \%$ of patients, while in 98.8 patients, these echocardiographic criteria were not reported. No reduction in vascular diameter was present in 16 patients, equivalent to $2.9 \%$ of patients, while this criterion was reported negatively in 224 patients, equivalent to $93.3 \%$ of patients. Mitral valve insufficiency of any severity was positive in 6 patients ( $2.5 \%$ of patients) and finally only 1 patient $(0.4 \%$ of patients) had pericardial effusion.

\section{Kawasaki diagnostic criteria}

Among the patient records in the present study, 128 patients met Kawasaki main criteria, 109 patients did not meet the diagnostic criteria and had 2 or 3 main criteria along with 3 complementary criteria with fever for more than 5 days and 4 patients They had echocardiographic criteria for the disease. The relative frequency of these patients was $53.11 \%, 45.22 \%$ and $1.67 \%$, respectively.

\section{Resistance and response to treatment}


Response to treatment in the present study was defined as cessation of fever up to 24 hours after the start of IVIG treatment with no recurrence, and resistance to treatment was defined as persistent fever in patients after 24 hours despite initiation of IVIG administration. However, 153 patients (63.5\%) showed appropriate response and 88 patients (36.5\%) showed resistance to treatment.

\section{The interval between the start of treatment and the onset of symptoms}

The mean time interval between the start of treatment and the onset of symptoms in the studied patients was 3.86 3. 6.86 days. This criterion also had no significant relationship with response to treatment with response to IVIG treatment in Kawasaki patients ( $p$-value $=0.11$ ).

\section{Footnote:}

[1] High power field of urine analysis by microscope

\section{Discussion}

Kawasaki disease (KD) is a common systemic vasculitis of unknown cause in early childhood that affects the skin, entire body mucosa, lymph nodes, meninges, intestine, liver, gallbladder, joints, genitourinary and heart, but the major damage of this multisystem vasculitis is mainly in the coronary arteries and nearly all epidemiologic studies show a higher susceptibility to KD in boys. Treatment with high doses of intravenous immunoglobulin (IVIG) can significantly prevent coronary aneurysms, although 10 to $20 \%$ of children do not respond to the IVIG treatment. Previous studies have shown that the ratio of neutrophils to lymphocytes after IVIG therapy in Kawasaki patients can predict resistance to IVIG therapy and coronary artery disease $1,8,11,12$.

Our study showed that there was no significant relationship between age and response to treatment in patients receiving IVIG. While the study of Egami et al. ${ }^{13}$ showed that age less than 6 months has a significant relationship with resistance to IVIG treatment. Kawamura et al. ${ }^{9}$ reported a similar result regarding to the significance of the relationship between age and resistance to treatment, but in their study, older age was associated with greater resistance to treatment. Three specific risk scores have been constructed by Japanese researchers; of these, the Kobayashi score is the most widely used and has high sensitivity and specificity. Unfortunately, application of these risk scores in non-Japanese populations do not appear to accurately identify all children at risk for IVIG resistance and CAA ) coronary artery aneurysm) ${ }^{12}$.

However, the results of our study are consistent with the Kobayashi study, and their study did not report a significant relationship between response to treatment and patients age ${ }^{14}$. The study by Hong et al. ${ }^{15}$ on children with KD showed that the patients with NLR less than 5 had significantly less age than patients who have NLRs greater than or equal to 5. Our study wasn't comparable to Hong's study in this respect. The NLR in our study had no significant relationship with the response to IVIG treatment. This is while the final p-value of our analysis was 0.058. But both Kee and Kawamura studies have reported a strong 
significant relationship between IVIG response ( $p$-value $<0.001)$ and NLR, so that NLR in refractory cases was reported significantly higher than in patients with appropriate treatment response.

Regarding patients' gender and its relationship with response to treatment, only the study of Kee et al. ${ }^{10}$ reported a significant relationship between male gender and resistance to treatment, and the other studies $9,13,14,16$ had similar results reporting that gender had no significant relationship with response to treatment. In our study, which had consistent results with the above articles $9,13,14,16$, the response to treatment was not significantly different between males and females.

Reactive protein C or CRP, one of the important markers of inflammation in the children, especially in rheumatic diseases and vasculitis ${ }^{17}$, had no significant difference between patients with appropriate response and cases with resistance to treatment in our study. However, in some studies $3,13,14,16$ this difference was significant and in others such as Kee et al., the difference was not significant ${ }^{10}$.

Raw neutrophil and lymphocyte count in the analysis of data obtained from the present study, had no significant difference between the two groups of patients with appropriate and resistant response. Previous studies, however, have reported a significant difference between neutrophil and lymphocyte counts in the two groups of patients with response and resistance to IVIG 9, 10,13-16. However, in our study, the level of white blood cells or leukocytes in the patients had a significant relationship with the response to IVIG treatment in Kawasaki patients, so that the number of blood leukocytes in patients who responded to IVIG treatment was significantly higher than the resistant to IVIG treatment patients. It must be noted that this finding is consistent with the study of Lee et al. ${ }^{16}$, but is contrary to the findings of other previous studies $9,10,13-15$.

In the present study, hypoalbuminemia was defined as less than $3 \mathrm{~g} / \mathrm{dl}$, which was selected based on the definitions in previous articles, but data analysis did not show a significant relationship between hypoalbuminemia and response to treatment in the present study. The finding of the article was contrary to the results of Kobayashi et al. ${ }^{14}$. But it was consistent with the findings of articles by Egami, Kawamura, and Lee et al. ${ }^{9,13,16}$.

The results of our study did not show a significant increase in the enzyme alanine transaminase or ALT, which was consistent with the study of Kee et al. ${ }^{10}$, but contrary to the findings of the other three studies of Egami, Kawamura and Kobayashi 9, 13,14. Of course, it must be noted that studies have not used the regulation of alanine enzyme levels based on age and comparison of increased state, and only they have reported an increase based on the results obtained and the use of Mann-Whitney $U$ test.

The assessment of hemoglobin and anemia, according to the adjustment based on the age of patients, was performed only in the study of Egami et al. and it was indicated that there was no significant relationship between the presence of anemia in patients and the response to IVIG treatment ${ }^{13}$. The present study's results were consistent with the results of Egami et al. study, and there was no significant difference between resistance and appropriate response to IVIG with anemia. 
The assessment of the number of leukocytes in the urine analysis of patients and its relationship with the response to treatment with IVIG, according to the literature researches were done so far, the assessment has not been done separately and the researchers in this study investigated this issue. The findings of the present study indicated that there was a significant difference between pyuria above 10 per HPF and response to treatment. In patients with leukocytes less than 10 or 10 per urinary HPF, the response to treatment is significantly higher than patients with urinary leukocytes more than 10 per urinary HPF. Even some patients who were hospitalized with fever, restlessness and pyuria were initially admitted to the nephrology ward with impression of urinary tract infection due to the presence of leukocytes in the urine. These patients later found the typical symptoms of Kawasaki disease and were therefore treated with diagnosis of Kawasaki disease ${ }^{12}$. This may highlight the importance of kidney or urinary tract involvement in Kawasaki disease. Of course, more and more extensive studies are needed in this regard. In fact, it can be concluded that the lower the rate of inflammation and renal involvement of patients (based on the presence of leukocytes in the urine), the more appropriate patients respond to treatment with IVIG.

The results of the present study did not show a significant relationship between thrombocytosis and response to treatment in this disease. Previous studies with the approach of absolute platelets count (regardless of the presence or absence of thrombocytosis and thrombocytopenia) have performed which have had contradictory results; Egami study reported a significant relationship between increase, Kawamura study a significant relationship between decrease and Kobayashi and Lee studies also reported a lack of significant relationship between platelet count and response to treatment $9,10,13,14,16$.

Finally, the results of the present study did not show a significant relationship between the mean time interval between the onset of symptoms and the start of treatment in patients responding to IVIG treatment. Previous studies have shown that there is a significant relationship between the time interval of the treatment starting and the onset of symptoms, but the results have been different due to the type of relationship (direct or inverse). Kawamura et al. reported this relationship was direct but Kobayashi and Lee's studies have reported this relationship reversely $9,14,16$.

\section{Conclusion}

The high blood leukocyte count and low urinary leukocyte count were directly related to response to treatment in Kawasaki patients and may be good predictors for response to treatment in Kawasaki patients receiving IVIG treatment.

\section{Declarations}

\section{Ethical Approval and Consent to participate}

In the present study, we used patient records and all patient information remained confidential. The implementation of this plan will be in full accordance with the ethical principles of the Helsinki 
Agreement ${ }^{18}$ in relation to working with the human sample. The present study has the ethics code IR.UMSU.REC.1398.257 registered in the ethics committee of Urmia University of Medical Sciences.

\section{Consent for publication}

The patients' legal guardians gave signed consent for publication.

\section{Availability of supporting data}

The data that support the findings of this study are available from the corresponding author upon reasonable request.

\section{Competing interests}

The authors have no conflicts of interest to declare.

\section{Funding}

The present study was sponsored by the Urmia University of Medical Sciences. There was no other organizational or governmental funding. The funding body did not have any role in the design of the study and collection, analysis, and interpretation of data, and in writing the manuscript.

\section{Authors' contributions}

R.F. and A.S participated in data collection, analysis and original draft writing. H.H. participated in supervision, study design, and final draft review.

\section{Acknowledgements}

We want to acknowledge all the healthcare providers of the Motahari Pediatric Center, particularly the personnel of the Immunology and Allergy ward.

\section{References}

1. Burns JC, Glode MP. Kawasaki syndrome. Lancet Aug 7-13. 2004;364(9433):533-44. doi:10.1016/S0140-6736(04)16814-1.

2. Narayanan SN, Ahamed MZ, Safia M. Cardiovascular involvement in Kawasaki disease. Indian Pediatr Sep. 2005;42(9):918-22. 
3. Ng YM, Sung RY, So LY, et al. Kawasaki disease in Hong Kong, 1994 to 2000. Hong Kong Med J Oct. 2005;11(5):331-5.

4. Krohn $\mathrm{C}$, Till H, Haraida S, et al. Multiple intestinal stenoses and peripheral gangrene: a combination of two rare surgical complications in a child with Kawasaki disease. J Pediatr Surg Apr. 2001;36(4):651-3. doi:10.1053/jpsu.2001.22312.

5. Baker AL, Lu M, Minich LL, et al. Associated symptoms in the ten days before diagnosis of Kawasaki disease. J Pediatr Apr. 2009;154(4):592-5 e2. doi:10.1016/j.jpeds.2008.10.006.

6. Burns JC, Mason WH, Glode MP, et al. Clinical and epidemiologic characteristics of patients referred for evaluation of possible Kawasaki disease. United States Multicenter Kawasaki Disease Study Group. J Pediatr. May 1991;118(5):680-6. doi:10.1016/s0022-3476(05)80026-5.

7. Cai Z, Zuo R, Liu Y. Characteristics of Kawasaki disease in older children. Clin Pediatr (Phila) Oct. 2011;50(10):952-6. doi:10.1177/0009922811409027.

8. McCrindle BW, Rowley AH, Newburger JW, et al. Diagnosis, Treatment, and Long-Term Management of Kawasaki Disease: A Scientific Statement for Health Professionals From the American Heart Association. Circulation Apr. 2017;25(17):e927-99. doi:10.1161/CIR.0000000000000484. 135 ) .

9. Kawamura Y, Takeshita S, Kanai T, Yoshida Y, Nonoyama S. The Combined Usefulness of the Neutrophil-to-Lymphocyte and Platelet-to-Lymphocyte Ratios in Predicting Intravenous Immunoglobulin Resistance with Kawasaki Disease. J Pediatr Nov. 2016;178:281-4 e1. doi:10.1016/j.jpeds.2016.07.035.

10. Ha KS, Lee J, Jang GY, et al. Value of neutrophil-lymphocyte ratio in predicting outcomes in Kawasaki disease. Am J Cardio/ Jul. 2015;15(2):301-6. doi:10.1016/j.amjcard.2015.04.021. 116 ) .

11. Zahorec R. Ratio of neutrophil to lymphocyte counts-rapid and simple parameter of systemic inflammation and stress in critically ill. Bratisl Lek Listy. 2001;102(1):5-14.

12. Kliegman RM. Nelson textbook of pediatrics. 21st edition. ed. Elsevier; 2019:pages cm.

13. Egami K, Muta $\mathrm{H}$, Ishii $\mathrm{M}$, et al. Prediction of resistance to intravenous immunoglobulin treatment in patients with Kawasaki disease. J Pediatr Aug. 2006;149(2):237-40. doi:10.1016/j.jpeds.2006.03.050.

14. Kobayashi T, Inoue $Y$, Takeuchi K, et al. Prediction of intravenous immunoglobulin unresponsiveness in patients with Kawasaki disease. Circulation. Jun 6 2006;113(22):2606-12. doi:10.1161/CIRCULATIONAHA.105.592865.

15. Cho HJ, Bak SY, Kim SY, et al. High neutrophil: lymphocyte ratio is associated with refractory Kawasaki disease. Pediatr Int Jun. 2017;59(6):669-74. doi:10.1111/ped.13240.

16. Lee SM, Lee JB, Go YB, Song HY, Lee BJ, Kwak JH. Prediction of resistance to standard intravenous immunoglobulin therapy in kawasaki disease. Korean Circ J Nov. 2014;44(6):415-22. doi:10.4070/kcj.2014.44.6.415.

17. Pepys MB, Hirschfield GM. C-reactive protein: a critical update. J Clin Invest Jun. 2003;111(12):1805-12. doi:10.1172/JCl18921. 
18. World Medical A. World Medical Association Declaration of Helsinki. Ethical principles for medical research involving human subjects. Bull World Health Organ. 2001;79(4):373-4.

\section{Tables}

Table 1 Comparison of means of quantitative variables in Kawasaki patients, according to the type of patient response to IVIG treatment. *neutrophil to lymphocyte ratio.

\begin{tabular}{|llll|}
\hline Variable & $\begin{array}{l}\text { Responsive to the } \\
\text { Treatment }\end{array}$ & $\begin{array}{l}\text { Resistant to the } \\
\text { Treatment }\end{array}$ & $\begin{array}{l}\text { P- } \\
\text { value }\end{array}$ \\
\hline Age (week) & $32.62 \pm 24.67$ & $38.75 \pm 26.35$ & 0.196 \\
\hline Time interval (day) & $6.86 \pm 3.79$ & $6.14 \pm 2.81$ & 0.110 \\
\hline Neutrophil count $\left(\times 10^{2} / \mathrm{mm}^{3}\right)$ & $60.99 \pm 17.36$ & $66.11 \pm 16.18$ & 0.275 \\
\hline $\begin{array}{l}\text { Lymphocyte count } \\
\left(\times 10^{2} / \mathrm{mm}^{3}\right)\end{array}$ & $35.46 \pm 33.8$ & $26.14 \pm 14.83$ & 0.142 \\
\hline $\mathrm{N} / \mathrm{L} \mathrm{ratio*}$ & $3.06 \pm 2.89$ & $4.00 \pm 3.51$ & 0.058 \\
\hline CRP $(\mathrm{mg} / \mathrm{L})$ & $40.79 \pm 26.33$ & $43.84 \pm 29.88$ & 0.163 \\
\hline Blood WBC $\left(/ \mathrm{mm}^{3}\right)$ & $14266.3 \pm 6291$ & $13450.3 \pm 4443.4$ & 0.013 \\
\hline
\end{tabular}

Table 2 Comparison of qualitative variables in Kawasaki patients, according to the type of patient response to IVIG treatment. 


\begin{tabular}{|c|c|c|c|c|}
\hline \multirow[t]{2}{*}{ Variable } & & \multicolumn{2}{|c|}{ Response to the treatment $(\mathrm{n})$} & \multirow[t]{2}{*}{ P-value } \\
\hline & & Responsive & Resistant & \\
\hline \multirow[t]{2}{*}{ Sex } & Male & 85 & 52 & \multirow[t]{2}{*}{0.80} \\
\hline & Female & 67 & 36 & \\
\hline \multirow[t]{2}{*}{ Serum Albumin } & $<3 \mathrm{gr} / \mathrm{dL}$ & 6 & 7 & \multirow[t]{2}{*}{0.18} \\
\hline & $\geq 3 \mathrm{gr} / \mathrm{dL}$ & 147 & 81 & \\
\hline \multirow[t]{2}{*}{ Anemia } & Positive & 111 & 71 & \multirow[t]{2}{*}{0.15} \\
\hline & Negative & 42 & 17 & \\
\hline \multirow[t]{2}{*}{ ALT } & Elevated & 25 & 21 & \multirow[t]{2}{*}{0.15} \\
\hline & Normal & 128 & 67 & \\
\hline \multirow[t]{2}{*}{ Platelets } & $\geq 450,000 / \mathrm{mm}^{3}$ & 60 & 31 & \multirow[t]{2}{*}{0.53} \\
\hline & $<450,000 / \mathrm{mm}^{3}$ & 93 & 57 & \\
\hline \multirow[t]{2}{*}{ Blood WBC } & $\geq 15,000 / \mathrm{mm}^{3}$ & 50 & 26 & \multirow[t]{2}{*}{0.25} \\
\hline & $<15,000 / \mathrm{mm}^{3}$ & 103 & 62 & \\
\hline \multirow[t]{2}{*}{ Urine leukocytes } & $\geq 10$ & 46 & 27 & \multirow[t]{2}{*}{0.01} \\
\hline & $<10 / \mathrm{HPF}$ & 61 & 107 & \\
\hline
\end{tabular}

